\title{
Fêtes et renouveau culturel
}

\section{Marie-Noëlle Ottino-Garanger}

\section{OpenEdition}

Journals

Édition électronique

URL : http://journals.openedition.org/jso/1610

DOI : $10.4000 /$ jso. 1610

ISSN : 1760-7256

\section{Éditeur}

Société des océanistes

\section{Édition imprimée}

Date de publication : 1 décembre 2001

Pagination : 193-200

ISSN : 0300-953x

\section{Référence électronique}

Marie-Noëlle Ottino-Garanger, "Fêtes et renouveau culturel », Journal de la Société des Océanistes [En ligne], 113 | Année 2001-2, mis en ligne le 27 mai 2008, consulté le 05 mai 2019. URL : http:// journals.openedition.org/jso/1610; DOI : 10.4000/jso.1610 


\section{Fêtes et renouveau culturel}

par

Marie-Noëlle OTTINO-GARANGER *

\section{RÉSUMÉ}

Les territoires excentrés de pays en pleine modernisation voient leur population diminuer, leur tradition petit à petit abandonnée; les plus jeunes ne portent plus de respect à la culture de leurs aînés ou, dans le meilleur des cas, se sentent très peu concernés par elle. À moins qu'elle ne retrouve un sens, qu'on lui porte attention, de l'extérieur en particulier, et que cette valeur redécouverte apporte de la vie, des ressources, de la fierté... C'est un des enjeux et des résultats du renouveau culturel qui passe par les grandes réjouissances que sont les festivals.

Mots-clés : Tradition, modernité, patrimoine, renouveau culturel, développement, identité, art, artisanat.

\section{Avant-propos}

La Société des Océanistes a lancé en 1999 un appel à contribution sur le thème « Folklorisation et cultures océaniennes : festivals, manifestations touristiques, marchandises folkloriques ». L'archipel des Marquises, à environ 1500 $\mathrm{km}$ de Tahiti, était alors en pleine préparation du Festival des arts des Marquises qui allait se dérouler sur l'île de Nuku Hiva fin décembre 1999, janvier 2000. Les sites archéologiques, que les associations de l'île avaient décidé de mettre en valeur comme cadre des festivités, allaient accueillir pour la première fois des délégations venant d'autres archipels du Pacifique, le tout étant inscrit au nombre des célébrations du passage à l'an 2000 organisées par la France et le ministère de la Culture.

\section{ABSTRACT}

Remote areas of developing countries suffer loss of population and of local tradition; the younger generation shows lack of respect or at best indifference to the culture of the elders. But traditional culture may find new meaning, stimulated perhaps by the interest of outsiders, leading its bearers to invest it with new life, resources, and pride. Such a revival has been an early result of the periodic Marquesan festivities, called festivals, over the past twenty years.

Key words : Tradition, modernity, identity, cultural heritage.

Cet article paraît donc bien après l'achèvement des festivités, alors que les Marquises, avec le soutien du Territoire de la Polynésie française, se préparent au prochain festival qui se tiendra en 2003 sur l'île de Hiva Oa.

Nous aurions pu compléter et actualiser certaines parties du texte mais nous avons préféré conserver sa valeur de témoignage. Le résultat aura été que les lieux ont séduit, les hommes se sont rencontrés et l'archipel s'est doté d'outils culturels lui permettant à juste titre de faire valoir la qualité et l'importance de son patrimoine, ceci afin d'obtenir les moyens de l'entretenir, mais aussi pour attirer à lui des ressources économiques dont la population a besoin.

Les Marquisiens renouent depuis près de vingt ans avec une tradition de plusieurs siècles : celle des « festivals » ou plutôt de la fête qui était

* Ethno-archéologue. 
également une trêve et une série de compétitions en honneur des vivants mais aussi des meilleurs d'entre les morts.

\section{Pour ou contre s'appuyer sur le passé}

Pour les habitants des îles qui vivent au quotidien l'érosion ou la transformation de leur culture, la protection de leur patrimoine naturel, artistique, oral... est perçue comme une façon d'exister, de maintenir une identité qui se traduit par un ensemble de particularités dans ces domaines précis et dans bien d'autres, allant de la langue aux habitudes alimentaires. Face à eux se trouvent ceux qui souhaitent atteindre l'image de modernisme que diffusent les récits des visiteurs et l'image des téléviseurs; ceci les opposent souvent à tout ce qui touche la culture - la danse mise à part, dans une certaine mesure. Pour eux, de telles préoccupations sont une entrave à la modernité et ils le manifestent par un rejet pur et simple ou une indifférence réelle à la question et un solide immobilisme.

Pour un petit nombre cependant, face aux enjeux du développement, et en particulier là où les atouts économiques sont rares, la préservation et la mise en valeur de certains pans de culture se présentent comme une réponse, un peu confuse encore par la multiplicité de ses applications, face à l'exode des familles, au manque de perspectives et de revenus, à la méconnaissance qu'ont les jeunes de leur univers mais aussi leur envie d'exister, d'en savoir plus sur le passé afin de mieux se connaître ${ }^{1}$. Pour ces petites communautés éloignées de centres économiques, accessibles cependant à une forme de tourisme «vert", c'est le principal atout en dehors de l'exotisme qui leur est accordé d'emblée, et le sens de l'accueil. C'est aussi un apport économique très concret, là où artisanat, pensions de famille et petits restaurants existent, ainsi qu'un échange humain et une vie, un dynamisme qui rythment l'année.

\section{Une prise de conscience de quelques-uns}

Engagées dans cette bataille de longue haleine et parce que ce moyen est perçu, parfois, comme le combat de la seule ou de la dernière chance, on trouve surtout des femmes dont l'ascendant est important, qu'elles exercent ou non une fonction officielle. Des voyages, ou séjours, en dehors de leur archipel leur ont fait prendre conscience de l'attrait de la différence aux yeux des gens de l'extérieur. Toutefois, la méconnaissance des cultures océaniennes est si grande, et si générale, le poids des clichés est tel, le peu de littérature disponible si pauvre ${ }^{2}$ que l'attente en matière culturelle de la part des visiteurs se solde par beaucoup d'ignorance. Nombreux sont ceux qui découvrent, par hasard, que ces îles ont un passé et qu'il n'est pas négligeable. Ils y sont venus pour un tout autre prétexte... entraînés au loin par le soleil, la mer, parfois le travail, et souvent de puissants mythes : le bon sauvage, la douce vahine, le guerrier farouche, un rien cannibale... ou un pèlerinage sur les pas d'écrivains, de peintres, de vedettes. Tout ceci pour aboutir au plus grand éclectisme lorsqu'il s'agit d'emporter «des souvenirs", ce qui ne facilite en rien l'orientation des artisans vers telle ou telle qualité de production ${ }^{3}$.

\section{Une longue tradition de fêtes associée à des struc- tures spécifiques à l'archipel}

Pour la première fois depuis son entrée dans les tourbillons de l'Histoire, à l'aube des Temps Modernes (1595), les Marquisiens, avec l'aide du Territoire et de l'État, s'ouvrent sur l'extérieur et vont découvrir concrètement, chez eux, certains des liens qui les unissent à d'autres cultures polynésiennes, dont des représentants des Cook et des Kiribati.

Accueillir lors d'immenses festivités (koika) ${ }^{4}$ des milliers d'hôtes, les habitants de l'archipel ont pratiqué cet art de longue date. Ce fut, probablement de tout temps, un des ciments de la vie sociale, la plus sûre démonstration du prestige des communautés. Pour cela, les vallées se lancèrent dans de véritables compétitions touchant les domaines les plus variés : joutes oratoires, sportives, chorégraphiques, musicales... Dans le même temps ce furent aussi de véritables défis architecturaux qui furent soutenus. Entre les $\mathrm{Xv}^{\mathrm{e}}$ et XVIII ${ }^{\mathrm{e}}$ siècles, R.C. Suggs (1961) observe, pour

1. C'est aussi parce que les métropoles où vont ces familles développent de graves problèmes, touchant principalement les jeunes exilés, que certains se mobilisent pour tenter de développer des activités.

2. Ceci est essentiellement dû au peu d'intérêt que manifestent bien des maisons d'édition à publier autre chose que de l'image pour touristes ; ceci change un peu grâce aux initiatives, au courage, de quelques-unes, plus petites, plus novatrices. Le Salon du livre d'Outre-mer, organisé par le ministère à Paris, et quelques initiatives en province, ont en cela un réel intérêt.

3. L'exposition « Kannibals et Vahinés. Imagerie des mers du Sud » qui ouvre ses portes conjointement au Musée des Arts d'Afrique et d'Océanie et au Musée de Chartres est éclairante à plus d'un titre à ce sujet.

4. Koika au nord de l'archipel, koina au sud : festivités dans la langue du groupe nord ou du groupe sud, bien qu'il faille apporter quelques nuances à ce découpage géographique. 
Nuku Hiva, la multiplication d'espaces communautaires très structurés à cet effet.

La motivation, stimulée par le chef, une ambiance de fête et le prestige ressortant de la participation à de tels travaux d'aménagement, sanctionnés par des motifs de tatouage spécifiques, posa probablement peu de problèmes dans le contexte traditionnel; la matière: bois et pierre, était abondante. Les roches employées, issues d'un volcanisme récent, sont éparpillées en quantités de blocs basaltiques dans les vallées. Pour la plupart de ceux mis en œuvre, leur poids varie toutefois du quintal à plusieurs tonnes, ce qui suppose une remarquable organisation et beaucoup de technique; s'y ajoutent les rares spécimens de roches taillées de Polynésie, le plus souvent des tufs volcaniques : ke'etu. Ces roches assez tendres servirent non seulement à sculpter des tikis - ces statues anthropomorphes massives relativement bien connues - mais surtout les dalles rectangulaires portant aussi le nom de ke'etu qui étaient implantées, de chant, en façade de la limite supérieure des paepae prestigieux (plate-forme lithique à deux niveaux). Elles soulignaient leur statut particulier d'un bandeau dont les teintes vont du brun rouge à l'ivoire orangé. Ces ke'etu furent parfois ornés de basreliefs : de petits anthropomorphes, le plus souvent, ou des damiers, parfois un lézard et des chevrons. Selon le plan habituel auquel obéissait l'architecture locale depuis les temps les plus reculés, un abri végétal soigné se dressait sur cette partie arrière. Il était destiné à l'habitation des membres de haut rang de la communauté (famille des chefs et prêtres). D'autres structures signalées, ou identifiées, de la même façon pouvaient abriter des lieux de réunion, servir de maison des hôtes ou des guerriers ou bien encore protéger ce qu'il fallait tenir à l'écart (marqué par un interdit très fort), ou ce que le clan avait de plus sacré, de plus précieux : des supports de divinités, des ornements, des reliques, des objets associés à la tradition et au culte, etc.

Les vastes ensembles ou places des fêtes (tohua ou taha koika), qui étaient conçus ou réaménagés lors de telles circonstances, s'étendaient à proximité de l'unité d'habitation d'un chef et étaient susceptibles d'accueillir, pour certains, des milliers de personnes. Parallèlement, il pou- vait en exister de quasi familiaux, ou « corporatifs », plus orientés vers des activités restreintes. Sur les dix hectares mis en valeur pour le Festival dans la vallée de Hatiheu, il existe trois places rectangulaires de ce type ; une représentative du dernier cas évoqué : Tei'ipoka $(58 \mathrm{~m} / 26 \mathrm{~m})$ et deux immenses: Tahakia qui mesure $155 \mathrm{~m}$ de long pour $42 \mathrm{~m}$ de large (fermé sur un de ses petits côtés par le paepae d'un chef légendaire de l'île : Keikaha nui) et, à moins de $200 \mathrm{~m}$ de là, Kamuihei, mesurant $142 \mathrm{~m}$ sur $38 \mathrm{~m}$. Si l'on déborde quelque peu de ce périmètre, se trouve un quatrième tohua: Hikoku'a, mesurant $124 \mathrm{~m}$ sur $45 \mathrm{~m}$. Ce ne sont pas les seuls tohua de la vallée ; il en existe encore trois autres de plus de $100 \mathrm{~m}$ de long. Tous furent des théâtres actifs de la vie de Hatiheu jusque dans le courant de la seconde moitié du XIX ${ }^{\mathrm{e}}$ siècle, époque où celle-ci comptait encore cinq clans de la grande famille Taipi. Cette branche cadette, avec son aînée Tei'i, se partageaient l'île par moitié.

Au cours des siècles, ces grands « champs de représentation » de la vie tant sacrée que profane, que furent pour chaque grand groupe humain de l'archipel ces vastes enclos entourés de paepae et de gradins, s'étaient structurés autour d'un vaste espace rectangulaire de plus en plus grand et de mieux en mieux aménagé. Ceci, non seulement afin de respecter l'ordre social et les prérogatives d'accueil, mais aussi pour répondre de la façon la plus judicieuse aux exigences d'un relief très accidenté dans des îles de petite dimension.

L'ensemble de ces défis que se lancèrent les clans de l'archipel (Ati ku'a, Ati papa, Etu oho, Hapa'a, Hema, Katu oho, Naiki, Mioi, Puhioho, Tai oa, Te ava aki, Te Uhi tua.... ${ }^{5}$ façonna non seulement ce paysage escarpé mais créa aussi un climat d'émulation artistique qui atteint, semble-t-il, des sommets au XVIII ${ }^{\mathrm{e}}$ siècle, époque probable où furent sculptés les grands tikis, de l'île de Hiva Oa par exemple. Parmi eux, dans les derniers témoins, il faut citer le grand tiki de bois provenant d'un me'ae ${ }^{6}$ de Atuona, conservé à présent au Musée de Tahiti et des îles; il mesure $2,29 \mathrm{~m}^{7}$. Quant à Taka'i'i, farouche vainqueur d'une terrible guerre menée sur la côte nord de Hiva Oa, le bloc de tuf dans lequel il fut taillé atteint trois mètres et la carrière d'où

5. Une centaine de clans, dont on se souvenait encore précisément ou qui comptaient encore de quelques centaines de personnes à quelques individus à la fin $\mathrm{du} \mathrm{XIX}^{\mathrm{e}}$ siècle.

6. Ce terme désigne l'endroit le plus sacré d'un groupe. Il ne s'agit pas forcément d'un lieu caractérisé par un aménagement architectural précis mais d'un endroit associé à un événement crucial où se déroulait, en très petit comité, les cérémonies religieuses réservées au saint des saints.

7. Un autre $t i k i$, provenant du même me'ae, est conservé au Bishop Museum à Hawaii. Les dimensions des têtes de pierre qui furent retrouvées au cours des travaux menés sur le méae I'ipona, dans la vallée de Puamau en 1991, varient entre 0,30m et $1 \mathrm{~m}$. $\mathrm{La}$ « sœur jumelle » de la plus grande d'entre elles, immortalisant celle de grands chefs ou guerriers qui furent offerts en victime à cet endroit, est à présent conservée à Berlin. Il fallut douze hommes, dit-on, pour la transporter jusqu'au bateau. 
il fut extrait fut déclarée tapu (interdite), afin qu'aucun autre bloc n'en soit plus jamais extrait. Il compte autour de lui, sur ce me'ae I'ipona, quatre autres tikis de facture remarquable dont deux frôlent les deux mètres, d'autres sont brisés. Il s'agit probablement de trois tikis monumentaux des vaincus. Il faut ajouter à cela dix têtes sculptées et quelques pétroglyphes ${ }^{8}$.

\section{Faire vivre le passé au présent : pourquoi, comment !}

Nuku Hiva, comme le veut la tradition, a lancé en 1999 un nouveau défi : celui d'accueillir les hôtes qu'elle attend dans deux anciens ensembles prestigieux remis en valeur. L'arbre ne pousse pas sans être solidement enraciné ; Nuku Hiva entend le montrer tout comme l'importance du leg dont elle est dépositaire afin de se poser, ainsi qu'à ceux qui lui rendront visite à cette occasion, la fameuse série de questions illustrée par Gauguin: "Qui sommes-nous? D'où venons-nous? Où allonsnous?»

Lucien Kimitete, conseiller-maire de l'île: haka'iki (chef) de Nuku Hiva, en frappant aux portes du troisième millénaire par cette interrogation donnant à chacune des journées un thème : savoir, conserver, transmettre... aime à rappeler à qui veut l'entendre :

« Tant de gens viennent ici pour connaître l'endroit où reposent Brel et Gauguin [...] pourtant les Marquises ne sont pas un cimetière! Nous sommes bien vivants et entendons le faire savoir haut et fort. Nous avons certes bien failli disparaître ; il n'y avait plus que 2094 habitants en 1926, aux Marquises, alors qu'autrefois certains disent que nous étions plus de 50000 . Chaque année, plusieurs peuples et langues disparaissent. Notre patrimoine, comme nous, a souffert. Il est en partie disséminé à travers le monde comme autant d'ambassadeurs ; ce qui reste est noyé sous la brousse ou détruit au fur et à mesure d'aménagements [...] Il est cher de venir et pourtant des gens arrivent du monde entier. Ils restent quelque temps et disent que c'est très beau, qu'il faut faire quelque chose pour protéger tout cela quand il en est encore temps. Certains essayent depuis des années, avec bien des difficultés. Des associations sont nées pour le protéger [...] et puis se fatiguent ! Nous sommes peu nombreux, loin, et tout est à inventer : protéger sans empêcher le développement, par exemple !».

C'est peut être là, l'un des tout premiers pas. Il n'est pas simple, car il est difficile de se rendre compte de la valeur de ce qui fait son quotidien. Si certains font des milliers de kilomètres pour le voir, les vallées qui veulent grandir aimeraient bien, ou ne résistent pas à la tentation de " pousser un peu ", de dégager tous ces cailloux et ces arbres, que ce soit plus simple et que cela ressemble un peu à ces images d'ailleurs, avec du ciment et des murs autour des maisons... pour «faire propre »!

Pour les Marquisiens d'un certain âge, les gens des vallées dont la vie consiste à accomplir des gestes traditionnels étroitement liés à la terre où ils vivent, et la mer qui la baigne, la série de questions est une sorte de mystère; elle fait rire : "Qui sommes-nous, nous qui sommes? Ce que nous sommes... Ce que nous faisons entre tai et uta (la mer et la montagne)! Des gens d'ici qui savons encore nommer tous ces endroits qui fourmillent de noms qui racontent la vallée, depuis les crêtes jusqu'à la côte, avec celui des familles qui y habitent, des poissons, des coquillages et des plantes dont on se sert... ou plutôt... ceux et celles que nous utilisons toujours car beaucoup nous échappent, à présent que nous n'allons plus beaucoup les chercher pour nous soigner, faire le tapa ou bâtir... Mais ils nous nourrissent encore un peu, moins que nos parents et plus que nos petits-enfants qui ne comprennent plus toujours les mots que nous employons, eux qui vivent à Tahiti ».

Voilà l'un des autres enjeux : savoir faire vivre, valoriser ceux qui savent encore... aussi modestes soient les gestes et les sujets. Prendre conscience du savoir qui disparaît, que l'on n'utilise plus, que personne ne recueille; se défier du savoir qui se transforme, par pudeur ou puritanisme et, parallèlement, de celui qui se crée, de toute pièce, parce qu'il flatte.

Voici quelques-unes des questions qui ne peuvent qu'immanquablement se poser à qui accompagne l'importante mutation de ces îles rattachées à l'un des rares pays du Pacifique où le revenu par habitant n'a pas fléchi grâce à une très forte intervention de l'État après le désengagement nucléaire. Sans en avoir pleinement conscience pour beaucoup, mais parce que nul n'échappe au courant créé par ses propres enfants, le Marquisien entre dans un monde élargi qui l'entraîne au-delà de ce qu'il souhaite pour lui-même. Sa réponse pour l'heure est de n'y pas trop penser et de profiter du moment présent jusqu'à ce que certains, par leur charisme, leur dynamisme, réveillent la belle qui dort avant la fête, les chants, les danses et les grands kaikai : une abondance de nourriture en rapport avec l'événement.

8. Takaii est le plus grand monolithe sculpté (2,63 m hors sol) de Polynésie, les moai de l'île de Pâques mis à part. 


\section{Qui porte l'événement ?}

Cette fois, contrairement au festival de 1989, les sculpteurs généralement porteurs de l'élan créatif sont peu présents. Uki Haïti et son frère avaient su les stimuler et les rassembler autour d'une nouvelle forme d'expression, à travers la pierre et un projet fédérateur ouvert aux jeunes, mais ils sont décédés. Si l'un d'entre eux - Tui Taupotini, le plus unanimement respecté - est président de l'organisation du festival, la plupart sont occupés à la réalisation de commandes pour un grand hôtel qui accompagne l'île dans le mouvement de développement orienté, par le Territoire, vers le tourisme.

La sculpture depuis son renouveau exprime la conscience culturelle de l'archipel ; Carol Ivory suit du reste dans son travail cette évolution. Cet artisanat, qui parfois touche à l'art, entraîne avec lui, de même que la chanson marquisienne, le réveil de plusieurs autres formes d'expressions artistiques dans lesquelles les Marquisiens se trouvent spontanément à l'aise et à travers lesquelles ils accèdent à une reconnaissance dépassant les limites de l'archipel; là aussi quelques jeunes universitaires se penchent sur la question. Pour cela il a fallu, et il faut encore, passer par une phase de production de "curios" : terme employé souvent pour désigner, avec un peu de mépris, certains pièces inspirées de la tradition et destinées à la vente. Celles-ci sont en fait le produit d'une libération, celle donnée par un outillage plus efficace que les baleines de parapluie et couteaux de leurs aînés. Si, parfois, la tentation est d'aller vite en besogne, la plupart ont la fierté de leur travail et ils mettent beaucoup de talent au service d'une vraie jubilation à créer un style chargé, « rococo ». Cette évolution répond à un amour spontané, ici, de l'accumulation qui est à mettre en corrélation probablement avec la longue expérience de privation. Si ces productions ont cet aspect « bâtard », c'est aussi en bonne partie par méconnaissance des objets anciens dont la plupart sont à l'étranger ou à Tahiti. En l'absence de musée local, il faut du reste saluer la volonté de l'évêque des Marquises de mettre à disposition des personnes soucieuses de les découvrir, des objets anciens présentés par la gérante de l'hôtel, déjà citée, qui s'intéresse à cette question depuis des années.

La façon dont est réduit, en quelques instants, à un tas de terre aux arbres éclatés un lieu ancien qui avait son charme et aurait pu abriter avec bonheur un aménagement moderne moins drastique, est tout autant le résultat du même man- que de référence d'images de qualité, accessibles et attrayantes pour l'opinion. Nous sommes ici devant un autre problème grave, celui de la compréhension de la modernité et de ses aménagements ${ }^{9}$ joint à la fascination pour les engins puissants : de la tronçonneuse aux éventuelles pelleteuses. Ce ne sont pas les films et feuilletons à « six sous » d'outre-Atlantique qui relèveront l'enjeu bien que des émissions s'ouvrent sur l'univers polynésien... Comment appliquer un jugement sévère à celui qui a si peu de choix pour former sa propre imagination, et son goût, alors même que le succès accordé à telle ou telle pièce ou projet médiocre, par certains acheteurs ou décideurs, ne peut le guider.

\section{Renouveau des formes d'expression}

Pourtant, l'art d'exprimer par signes, symboles, images (que l'on appelle ici tiki), des formes ou ce que l'âme ressent fut, probablement de tout temps, un talent longtemps entretenu avec soin. Il s'appliqua à toutes sortes de supports, de l'inerte au vivant, des pétroglyphes aux tatouages, de la sculpture à l'architecture.

Il se développa de façon remarquable dans quelques archipels plus que d'autres, telles la Nouvelle-Zélande, Samoa, les Cook et les Australes mais aussi les Marquises où il faillit disparaître au cours du siècle qui s'achève. Quelques familles continuèrent de pratiquer la sculpture sur bois, confectionnaient encore un peu de tapa, taillaient des pilons de pierre ou des leurres à bonite en nacre pour leurs hameçons. Par contre, l'art du façonnage de l'écaille ou de l'ivoire marin, des coquillages, des peaux de requins ou des plumes, des tambours aux prestigieuses parures, avaient totalement disparu.

Les retrouvailles avec la danse traditionnelle ou du moins trois qui avaient survécu sur les soixante-dix-sept dont le nom avait été conservé, furent célébrées à Tahiti en 1985 lors d'une des rares occasions où l'archipel reçut la possibilité de se rendre aux grandes fêtes de juillet, à Tahiti. Marlo Brando invita quelques membres de la troupe de l'île de Ua Pou à cette occasion. C'était, avec le maintien de la langue, une consécration des efforts du Motu Haka o te Henua 'Enana : l'une des plus anciennes associations, à la fois culturelle et artisanale, du Territoire, ces deux activités étant pratiquement indissociables. Il allait donner naissance au festival des Marquises et soutenir l'effort de création des groupes de danses et des artisans. 
C'est ainsi que resurgit pas à pas, par le biais de ces nouveaux défis mobilisateurs que furent les grandes fêtes communautaires, une part de l'âme d'un archipel; la forme peut décevoir, l'effet sur le fond n'en est pas moins là !

\section{La difficulté à se réconcilier avec les paroles du passé}

La volonté de sauvegarde de la tradition orale se heurta, quant à elle, à de la méfiance et un grand désintérêt pour la parole des vieux. La principale préoccupation sur le sujet, en dehors de questions religieuses, était l'usage qui pouvait être fait d'un patrimoine familial qui ne se transmettait, traditionnellement, que dans des conditions garantissant à un groupe précis une part de savoir et donc de pouvoir. Avec sa disparition, la question ne se pose même plus! Nombre d'anciens avaient conscience qu'avec eux disparaîtraient des pans entiers de mémoire ; leur prêter une oreille attentive était un bonheur dont on les priva par indifférence et manque crucial de moyens. L'Église catholique aida la langue à se maintenir, mais il aurait fallu enregistrer systématiquement les anciens des vallées et soutenir ceux qui voulaient bien s'y consacrer. Il fallait aider au long travail de transcription de ces bandes si fragiles. Il en allait de la sauvegarde de connaissances multiples, des plus goûtées : les légendes, aux plus précieuses : savoir technique, médicinal... jusqu'aux plus humbles. Toute une sagesse liée au quotidien, à l'usage de la mer, à la culture vivrière, au mouvement des étoiles, au nom des choses et des lieux qui disparaît de plus en plus vite et irrémédiablement. Une petite part fut préservée par des familles, des missionnaires, des visiteurs et chercheurs de passage. Une autre tente de l'être grâce aux efforts de quelques-uns ; c'était un des objectifs du festival mais l'on manqua de forces vives et de moyens. Le cœur y est encore néanmoins, pour un certain nombre, et le Territoire aide à la création d'une académie marquisienne à l'image de l'académie tahitienne. Puisse-t-elle ne pas exclure mais au contraire valoriser la parole de tous!

\section{L'aventure des festivals marquisiens}

C'est en 1987, puis en 1989, que se déroulèrent les tout premiers festivals, à $\mathrm{Ua}$ Pou tout d'abord, puis Nuku Hiva. Rendez-vous fut alors pris pour que se renouvellent ces échanges, les plus petites îles de l'archipel: Fatu Hiva, Tahuata et Ua Huka se rendant chez les plus peuplées: Ua Pou, Nuku Hiva et Hiva Oa.
Parallèlement, s'organisèrent d'autres rendezvous importants dont le concours des sculpteurs de Ua Huka autour de thèmes traditionnels : casse-têtes, étriers d'échasses et éventails, cette fois-ci. D'année en année, le souci d'authenticité et la qualité s'y développent; ceci va de pair avec la création, en 1985 sur cette même île, du premier musée municipal de l'archipel. Il doit son existence et son développement à la confiance faite, par le maire et les familles de l'île, au sculpteur qui en est le conservateur et bénéficie d'un poste à plein temps. Que de postes pourraient ainsi être créés pour la protection et l'entretien ou la mise en valeur du patrimoine. L'intérêt que ce merveilleux artisan porte à sa culture, et son dévouement, ont abouti à une réalisation remarquable soutenue par le Musée de Tahiti et des îles, pour la constitution des vitrines, enrichies grâce aux dépôts des habitants et une surveillance du patrimoine local. Pour les nombreuses pièces dont il n'existe plus d'exemples dans l'archipel, il a été fait appel à la photographie et au talent du sculpteur afin d'offrir aux Marquisiens, et aux visiteurs, une image vivante de ce que fut la culture d'autrefois: une initiative exemplaire.

Autre initiative décisive : celle de Hiva Oa, en 1991, qui fut la première à vouloir associer la restauration de sites prestigieux à la perspective d'un festival afin de profiter de l'élan suscité par celui-ci auprès des autorités. La vallée de Hatiheu, à Nuku Hiva, autour d'Yvonne Katupa, avait été précurseur en la matière en restaurant, avec toute la population, une grande partie du tohua Hikoku'a pour y accueillir les touristes de passage. L'idée, sa réussite, firent leur chemin ; c'est ainsi que furent restaurés sous la direction d'archéologues, par une main d'œuvre de jeunes et d'adultes bénéficiant d'un dispositif d'emploi soutenu par l'État et le Territoire, deux sites anciens majeurs pour l'ensemble de l'archipel : Ta'aoa et Puamau, respectivement au sud-ouest et au nord-est de l'île de Hiva Oa.

D'autres fêtes mémorables ponctuent l'intervalle de quelques années entre chaque festival, ainsi la célébration du $150^{\mathrm{ème}}$ anniversaire de la naissance de Paul Gauguin (en juin 1998, à Atuona, Hiva $\mathrm{Oa}$ ) à laquelle participèrent près d'une centaine de membres de la famille du peintre, venus pour la plupart de Scandinavie, à l'invitation du paquebot du même nom. Elles sont autant de répétitions où se rodent aussi bien des pièces de sculpture que des danses inspirées des pas et des rythmes traditionnels. Pour tous ces visiteurs faisant escale, quelques heures ou quelques jours, dans les îles, c'est un étonnement souvent, un ravissement parfois. Ces émotions 
sont suscitées aussi bien par l'écrin montagneux, très sombre et impressionnant, les baies, la végétation, que les personnes elles-mêmes, leur allure, leur visage, leur comportement, leurs chants et leurs danses...

\section{Du contact avec l'étrange}

"Faire la curiosité »: voici une expression charmante. N'est-ce pas une des fonctions cachées de la fête, en dehors du "faire valoir », tout autant qu'une des activités du touriste? Sait-il qu'il est lui-même du reste sujet d'observation et parfois occasion de changements?

Qui aura le plus regardé l'autre au terme de ces fêtes ? Probablement le Marquisien. Qui est le plus exotique par sa tenue? Souvent le touriste! Autant d'observations déroutantes allant en s'accumulant. L'accueil, dont divers comités associatifs ont la responsabilité, est en ce domaine un des premiers touchés : la main tendue au débarquement que l'on refuse (pour des raisons d'hygiène expliquent les grandes agences en contact avec la clientèle des paquebots), l'habituel collier, souvent très odorant et composé aux Marquises non seulement de fleurs mais aussi de nombreuses plantes très parfumées, qui n'est pas toujours accueilli avec enthousiasme par les touristes indisposés ou craignant de se tacher... Peu de choses mais qui s'ajoutent et provoquent assez vite un désintérêt pour ce genre de visites si elles ne sont pas accompagnées d'une véritable fête. Il est arrivé que les artisans de Nuku Hiva aient accepté de présenter les fruits de leur artisanat mais ne les aient pas mis en vente, les réservant à Tahiti, ce qui déroute les riches visiteurs de gros paquebots qui ne parvenaient pas à comprendre ces artisans. L'île voisine de Ua Huka s'organise tout autrement. Depuis déjà un certain temps, il y existe, par vallée, des coopératives d'artisans, hommes et femmes, qui veillent à maintenir une offre, même faible, et à contrôler en même temps la qualité et les prix.

Les produits offerts et les prix pratiqués sont en effet une des sources de surprises. La présentation, la signification, autant de questions que les Marquisiens ne se posent pas. Le franc pacifique et la façon d'estimer le travail en fonction des besoins : un produit ou un engin de travail, aboutissent souvent à des estimations curieuses, souvent élevées par rapport à ce que l'étranger en croisière a pu rencontrer dans les pays qu'il vient de quitter : l'Amérique latine souvent, et ce qu'il peut estimer, lui-même, de sa propre expérience... ailleurs.

\section{Ceux grâce à qui, sur le terrain, de telles aventures sont possibles}

Dans ce décor, brossé à grands traits, il faut évoquer les personnes qui travaillent pour que de tels paris puissent tenir. Autant organiser de gigantesques festins dans l'enthousiasme collectif est aisé à tous, ici, autant assurer au jour le jour des tâches répétitives et peu valorisantes est une gageure. Il faut sans relâche raviver l'intérêt, varier les occupations tout en les coordonnant afin que toutes aboutissent au résultat escompté au jour des festivités. Ceci suppose d'être en permanence sur la brèche pour avancer le dégagement de la brousse... et des dossiers, la collecte des matériaux, le dégagement des structures, la remise en place de blocs rocheux souvent très lourds, l'entretien d'un matériel mis à rude épreuve, prévenir les accidents, relever le moral abattu par le temps, la paie qui n'arrive pas ou la lenteur de certaines tâches, etc. Une entreprise de très longue haleine qui rejoint celle des personnes qui se battent pour donner à des vallées, encore peu nombreuses, un atout de qualité sur le plan touristique et culturel.

Il faut pour cela accomplir un travail de fond, régulier, sur tout un ensemble de données, afin d'obtenir une harmonie entre développement et aménagement «traditionnel ». Il faut gagner la confiance, inspirer l'envie de suivre, faire preuve d'autorité tout en ménageant les individualités, soutenir les initiatives, éviter les découragements... Y arrivent ceux dont l'image est respectée, mais même ainsi, il faut sans arrêt être présent sur le front de l'érosion, et parfois de la malveillance, pour éviter ou colmater les brèches irréparables faites à coup d'ignorance ou de paresse, de tronçonneuse ou de bull, par gloriole, méconnaissance ou jalousie...

Une fois franchies toutes ces étapes, après avoir su galvaniser la population, la fête peut alors commencer! La fête où se manifestent les dons artistiques de tout l'archipel et où ceux-ci s'affinent en se côtoyant; où ils s'élargissent et s'enrichissent de l'expérience des uns et des autres. Elle transcende le quotidien, elle stimule un vaste champ d'activités et pique au vif la créativité. C'est alors un merveilleux instrument réinventé pour redonner la vie au talent, le valoriser face à d'autres influences qui démotivent, valoriser ce qui est bien fait, fait avec amour, les gestes simples parfois mais qui valorisent un pays pour pouvoir y vivre. 


\section{RÉFÉRENCES}

Steinen K. von Den, 1999. Pohu, in Mythes marquisiens. Te hakatu tumu o te ati 'enana, Vol. 3, Haere Po no Tahiti.
SugGs R.C., 1961. Archaeology of Nuku Hiva, Marquesas Islands, French Polynesia, Anthropological Papers of the American Museum of Natural History, $\mathrm{n}^{\circ} 49$ (1), New York, American Museum of Natural History. 